Classification

Physics Abstracts

$42.55 \mathrm{P}$

\title{
Analyse des bruits blancs optique et électrique d'une tête d'émission laser à réaction répartie en présence d'une réinjection optique
}

\author{
P. Signoret, B. Orsal, J.M. Peransin et R. Alabedra \\ Centre d'Electronique de Montpellier (CNRS URA 391), Université Montpellier II, Sciences et \\ Techniques du Languedoc, 34095 Montpellier Cedex, France
}

(Reçu le 8 juillet 1994, révisé le 12 septembre 1994, accepté le 12 septembre 1994)

\begin{abstract}
Résumé. - Les performances, et tout particulièrement les bruits optique et électrique, d'une tête d'émission laser à semi-conducteur à réaction répartie (DFB), émettant à la longueur d'onde de 1,55 micromètres, sont étudiés. Cette caractérisation par le bruit permet de mettre en évidence et de distinguer deux phénomènes parasites de réinjection optique : le bruit de partition et le processus de compétition modale. Le lien étroit existant entre les densités spectrales de bruit électrique et de bruit optique est clairement montré. Cette propriété est illustrée par la corrélation entre ces deux types de bruit. Le bruit électrique permet ainsi une caractérisation fine des lasers à semi- conducteur, tout en évitant la génération de réflexions parasites issues de tout montage optique.
\end{abstract}

\begin{abstract}
The performances, and particularly the optical-electrical noise behaviour, of semiconductor distributed feedback (DFB) laser diodes emitting at 1.55 micrometers are studied. This noise characterization allows to bring to the fore and to distinguish two optical parasitic effects: partition noise and mode competition. The tight link between electrical and optical spectral densities is shown and strengthened by the measurement of the correlation function. The electrical noise can so be used for an accurate characterization of laser diodes, without any optics which might introduce undesired optical feedback.
\end{abstract}

\section{Introduction}

Les fluctuations d'intensité dans la lumière émise par une diode laser imposent une limite ultime aux performances de tout système de communication optique. Le travail présenté ici consiste en l'analyse de ces fluctuations, ainsi que du bruit électrique de terminaison (TEN), en présence d'une réinjection parasite optique. Cette étude concerne les émetteurs laser à réaction répartie (laser DFB) et est réalisée dans une plage de moyenne fréquence : $10 \mathrm{kHz}-500 \mathrm{kHz}$. Un modèle théorique fondé sur les équations d'évolution se rapportant aux populations d'électrons et de photons est également présenté dans ce travail. 


\section{Structure du laser DFB}

L'émetteur utilisé dans cette étude présente une couche guide d'onde à base de matériau quaternaire et à réaction répartie le long de la cavité, adjacente à la zone active, et élaborée sur un substrat d'InP : figure 1.

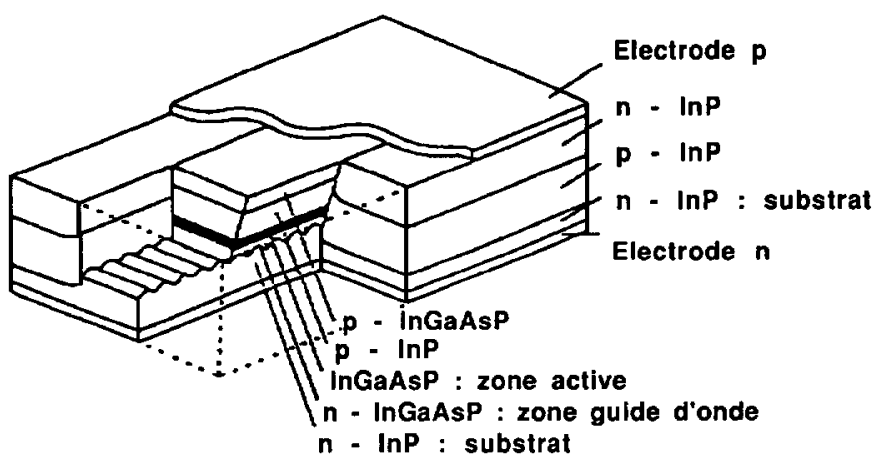

Fig. 1. - Représentation schématique d'un laser DFB à hétérostructure enterrée InGaAsP/InP. [Schematic structure of an InGaAsP/InP buried DFB heterojonction.]

Cette structure présente en outre une double homojonction latérale à base d'InP qui assure un excellent confinement des porteurs dans l'hétérojonction émettrice [1].

Cet émetteur fonctionne à la longueur d'onde de $1,55 \mu \mathrm{m}$.

\section{Caractérisation statique}

2.1. Caractéristique courant-tension : $I_{\text {Laser }}\left(V_{\text {Laser }}\right)$. - L'évolution, mesurée à $20^{\circ} \mathrm{C}$, du courant $I_{\text {Laser }}$ traversant la jonction laser en fonction de la tension $V_{\text {Laser }}$ aux bornes de la structure est donnée figure 2 .

Nous remarquons trois zones bien différenciées :

- la zone de très faible courant, jusqu'à environ $0,1 \mu \mathrm{A}$, dominée par les courants de fuite des diodes latérales,

- la zone où $I_{\text {Laser }}$ croît rapidement avec $V_{\text {Laser }}$. ce courant est dû principalement à la recombinaison thermique : recombinaisons par les centres recombinants, processus de génération-recombinaison, . [1],

- la région de saturation au delà de $V_{\text {Laser }} \approx 0,95 \mathrm{~V}$ : la zone d'inflexion, qui apparaît très nettement dans la caractéristique au voisinage de cette tension, correspond à la transition du régime de superluminescence vers le régime d'émission stimulée. Cette inflexion se situe principalement au seuil, $I_{\text {seuil }} \approx 19,5 \mu \mathrm{A}$, et traduit ainsi l'apparition du régime laser.

\subsection{CaRACTÉRISTIQUe RÉSISTANCE DIFFÉRENTIELle-COURANT INJECTÉ $: R_{\mathrm{d}}\left(I_{\text {Laser }}\right)$}

La connaissance de la résistance différentielle $R_{\mathrm{d}}$ est essentielle afin de mesurer correctement la densité spectrale de bruit électrique. 


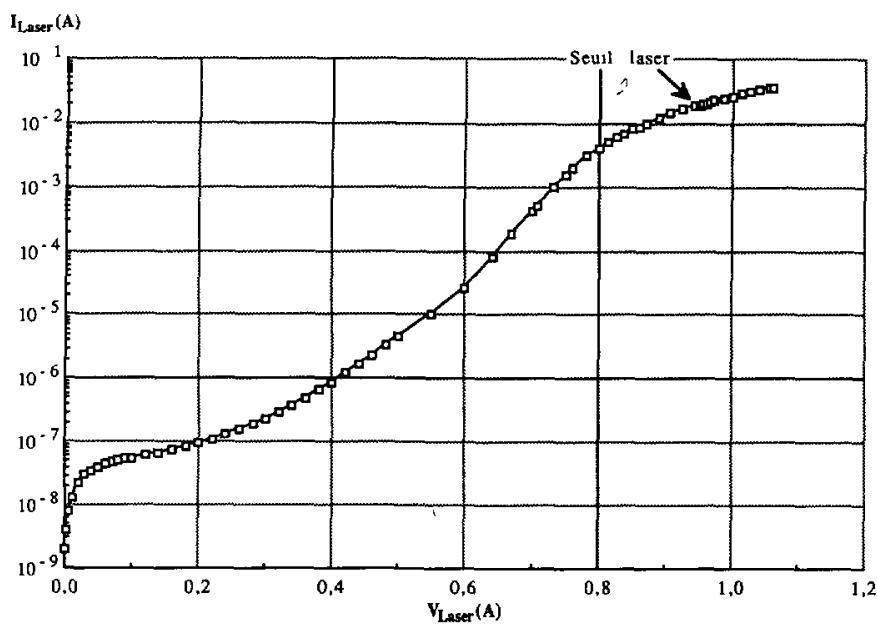

Fig. 2. - Caractéristique courant-tension du laser DFB étudié. [Static characterization of the DFB laser: current versus voltage.]

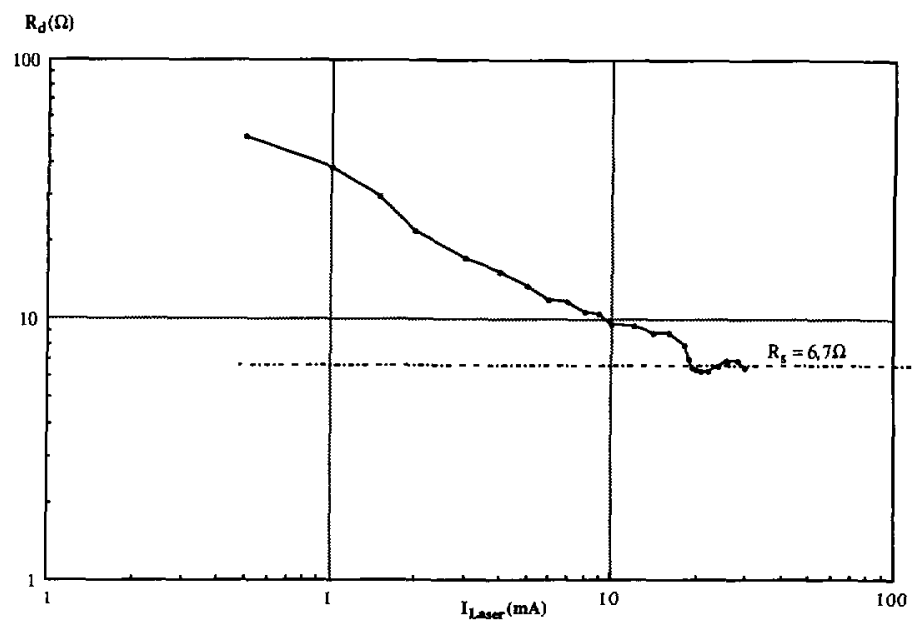

Fig. 3. - Résistance différentielle du laser DFB fonction du courant injecté.

[Differential resistance of the DFB laser versus laser current.]

L'évolution avec le courant laser de $R_{\mathrm{d}}$ est obtenue à partir de la caractéristique $I_{\text {Laser }}\left(V_{\text {Laser }}\right)$ précédente, par simple rapport de variations élémentaires, et est représentée figure 3 au voisinage du seuil laser.

En deçà du seuil, la résistance $R_{\mathrm{d}}$ évolue approximativement en $1 / I$ lorsque le courant injecté augmente ; dans cette zone prédominent les courants de génération-recombinaison thermique. Au delà, le courant et donc les recombinaisons saturent : $R_{\mathrm{d}}$ tend vers la résistance série $R_{\mathrm{s}}$ correspondant à la résistance des contacts et des différentes couches de la diode laser ; $R_{\mathrm{s}} \approx 6,5 \Omega$ pour le composant étudié. 


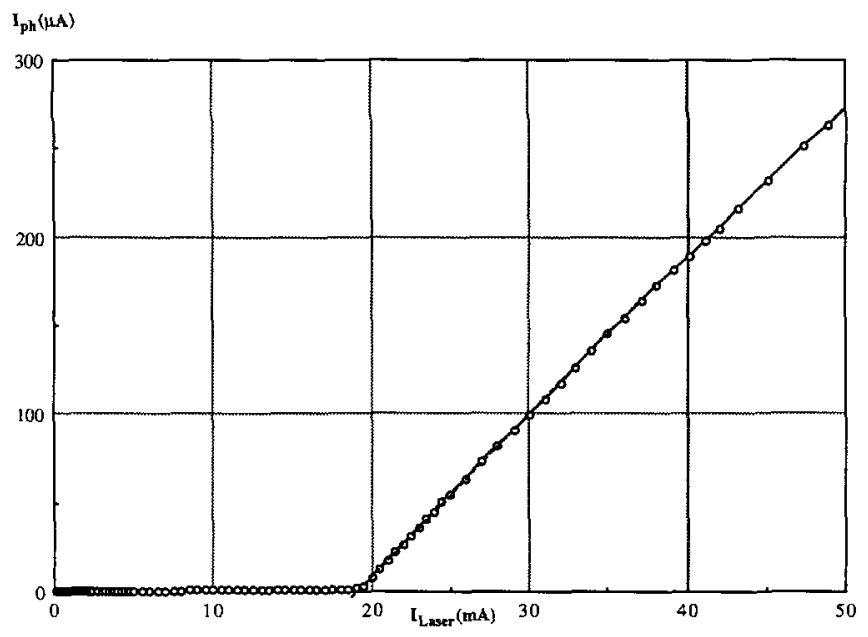

Fig. 4. - Evolution du photocourant détecté en fonction du courant laser.

[Detected photocurrent as a fonction of the injected current.]

La cassure observée dans la caractéristique reflète le point d'inflexion commenté précédemment : elle traduit l'apparition de l'émission laser.

2.3. Caractéristique puissance optique-Courant injecté : $P_{\text {opt }}\left(I_{\text {Laser }}\right)$. - La caractéristique $I_{\mathrm{ph}}\left(I_{\text {Laser }}\right)$, où $I_{\mathrm{ph}}$ désigne le photocourant détecté par une photodiode de contrôle interne à la tête optique, est donnée sur la figure 4 .

On distingue, comme usuellement, trois zones [2] : zone de faible croissance, "coude" et enfin évolution linéaire en régime laser.

Sur cette courbe, on constate que le passage du régime de diode électroluminescente au régime laser est très rapide : ceci est lié à la faible fraction $\beta$ d'émission spontanée du laser DFB [1].

De plus le courant de seuil mesuré est relativement faible : $I_{\text {seuil }} \approx 19,5 \mathrm{~mA}$ à $20^{\circ} \mathrm{C}$, c'est une des propriétés qui font l'intérêt de la structure laser DFB.

La puissance optique émise par la diode laser est déduite du photocourant détecté par la relation classique : $P_{\mathrm{opt}}=\frac{I_{\mathrm{ph}}}{\sigma}$ où $\sigma$ désigne la sensibilité apparente du photodétecteur. Dans le cas présent : $\sigma \approx 0,08 \mathrm{~A} / \mathrm{W}$.

\section{Analyse spectrale}

3.1. Présentation des deux Configurations de mesure. - En situation optique $(\alpha)$, schématisée sur la figure 5, la fibre optique placée en vis à vis d'une face émissive de la diode laser présente un poli à une extrémité. Celui-ci induit des réflexions optiques qui sont amplifiées dans la cavité laser ; la lumière réinjectée dans la zone active est en effet réfléchie à l'autre extrémité de la cavité laser, côté photodiode de contrôle, où la face de sortie est très réfléchissante $(R \approx 0,9)$. Cette lumière va donc générer un ou des modes optiques parasites qui, sous certaines conditions, auront suffisamment de gain pour être en compétition avec le mode de Bragg propre à la structure DFB. 


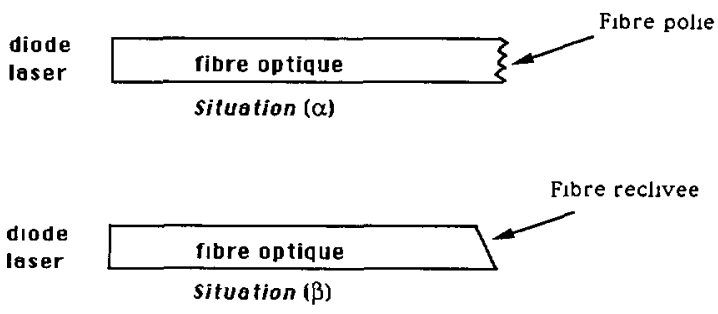

Fig. 5. - Schéma des deux situations de mesure.

[Schematic representation of the two optical configurations.]

En configuration $(\beta)$, le clivage en extrémité de la fibre optique a été amélioré. Les réflexions externes sont réduites mais non supprimées pour autant : nous sommes en effet toujours en présence d'une interface fibre/air. De plus, inhérent à la tête optique, se trouve une lentille de couplage positionnée entre l'émetteur et la fibre amorce et pouvant être source de réflexions vers la cavité laser.

3.2. Spectres optiques. - Sur la figure 6 sont donnés les spectres obtenus en situations $(\alpha)$ et $(\beta)$ pour des courants d'injection voisins de cinq fois le courant de seuil. Nous constatons sur la figure 6a un éclatement ("splitting") du spectre : il y a apparition de sous-modes. Un tel comportement spectral a été observé et modélisé par Lenstra [3] : tout phénomène de réinjection optique induit des changements de fréquence, un élargissement de la raie spectrale du laser et une compétition modale entre les différents modes issus de la "cavité externe". Qui plus est, un tel processus entraîne un effondrement de la cohérence optique. L'émetteur laser caractérisé présente donc, en configuration $(\alpha)$, un caractère multimodal : ce type de comportement survient lorsque la répartition de l'énergie des différents modes qui composent la puissance lumineuse de sortie fluctue considérablement [4].

En configuration $(\beta)$, nous remarquons qu'un clivage correct en extrémité de fibre améliore très nettement la largeur de raie spectrale : figure $6 \mathrm{~b}$. Le laser DFB est probablement moins perturbé par les réflexions parasites, le mode de Bragg devenant prépondérant. On peut pratiquement affirmer à la seule vue de ce spectre que le laser a, sous forte polarisation, un comportement quasi-monomodal.

\section{Etude des densités spectrales de bruit blanc}

La reproductibilité et la stabilité des caractéristiques statiques nous permettent de poursuivre l'étude de la diode laser DFB.

Nous avons étudié expérimentalement, en présence d'une réinjection optique, les densités spectrales de bruit électrique et optique de la tête laser à réaction répartie, dans une plage de moyenne fréquence $(10 \mathrm{kHz}<f<500 \mathrm{kHz})$.

4.1. Montage expérimental. - Ce montage, décrit dans [2], comprend, outre l'élément clé, c'est à dire l'analyseur FFT, deux chaînes d'amplification faible bruit :

- un amplificateur de tension connecté en parallèle sur la diode laser ; nous mesurons par cette voie les fluctuations $S_{V_{d}}$ de la tension aux bornes de l'émetteur DFB.

- un amplificateur trans-impédance de gain variable, placé en série avec le photodétecteur de contrôle, et nous permettant de mesurer les fluctuations $S_{I_{\mathrm{ph}}}$ du photocourant détecté. 


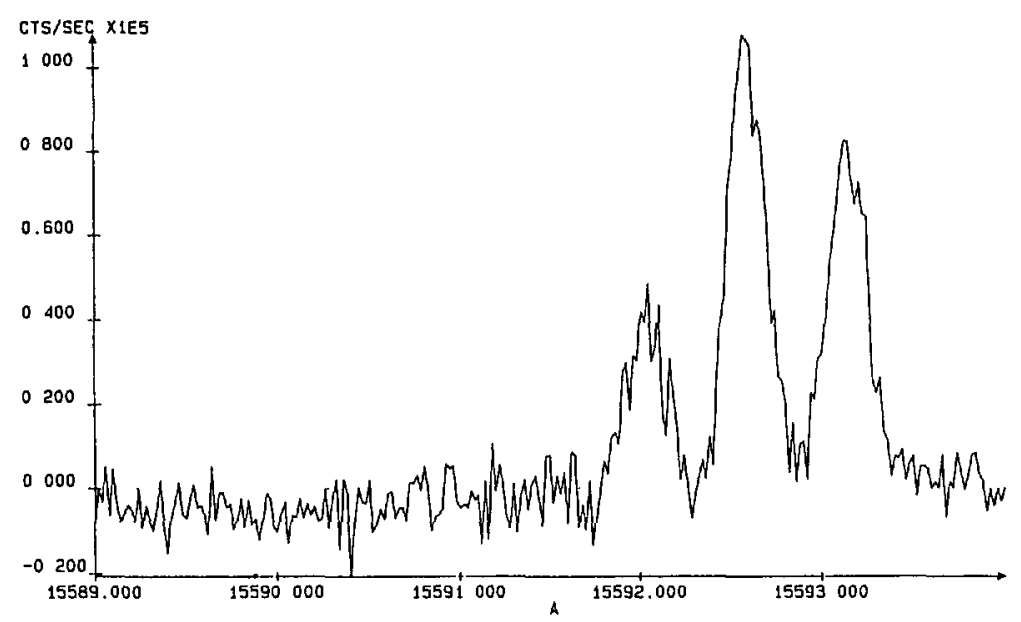

a)

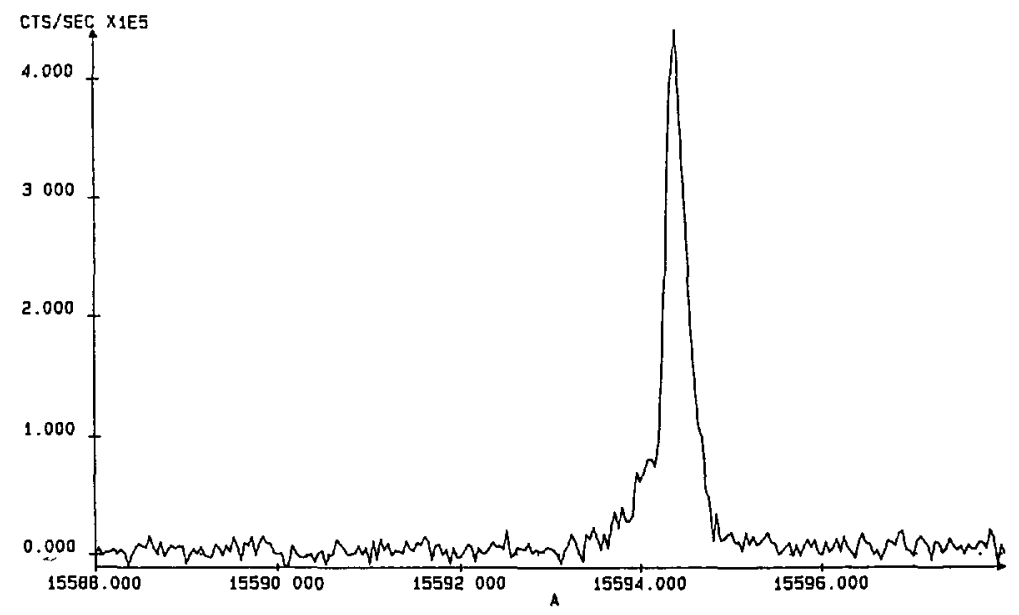

b)

Fig. 6. - Spectres optiques obtenus dans les deux configurations pour $I_{\text {Laser }} / I_{\text {seuil }} \approx 5$.

[Optical spectra in both configurations, for $I_{\text {Laser }} / I_{\text {seuil }} \approx 5$.]

\subsection{RÉSULTATS EXPÉRIMENTAUX À $200 \mathrm{KHz}$}

4.2.1. Spectres en fréquence. - Sur les figures $7 \mathrm{a}$ et $7 \mathrm{~b}$ sont donnés les spectres de bruit optique, obtenus au voisinage du seuil laser, respectivement en configuration optique $(\alpha)$ et $(\beta)$. L'allure est tout à fait "classique" : décroissance en $1 / f$ jusqu'à une fréquence de quelques $\mathrm{kHz}$, suivie d'un plateau de bruit blanc. La décroissance observée au-delà est simplement due à la fréquence de coupure de l'amplificateur utilisé, comprise entre 100 et $200 \mathrm{kHz}$.

En configuration $(\alpha)$, le niveau mesuré est toutefois relativement élevé : nous notons un écart d'environ deux décades entre les niveaux des deux plateaux de bruit blanc. Un tel comportement a été observé par plusieurs auteurs [5-7], qui ont bien montré que tout phénomène de réinjection parasite entraîne une remontée considérable du niveau de bruit, un élargissement de la raie spectrale et également une déviation en fréquence du spectre d'émission ("frequency chirp"). Ces événements sont donc étroitement liés. 


$$
S_{I p h}\left(A^{2} / H z\right)
$$

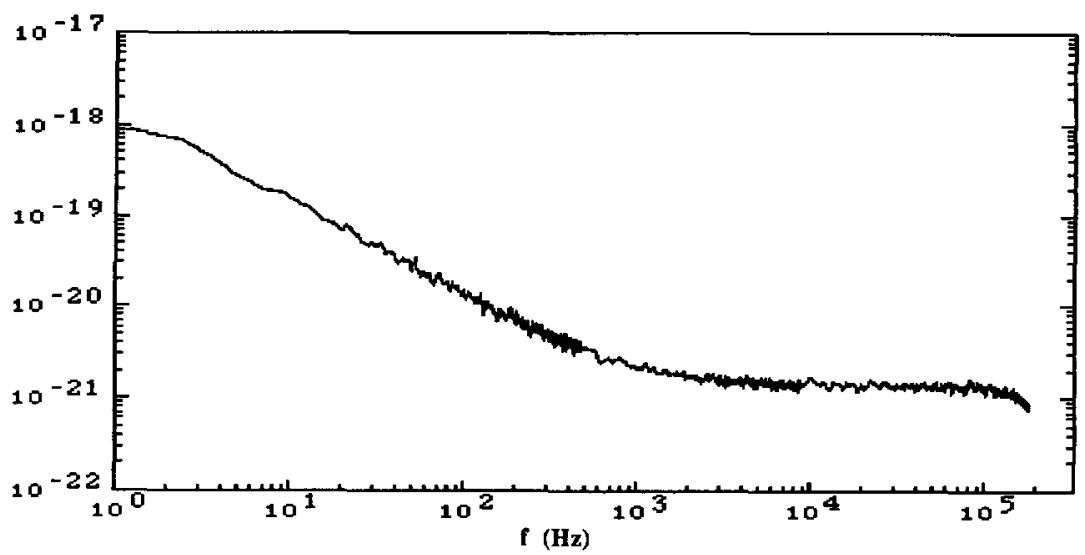

a)

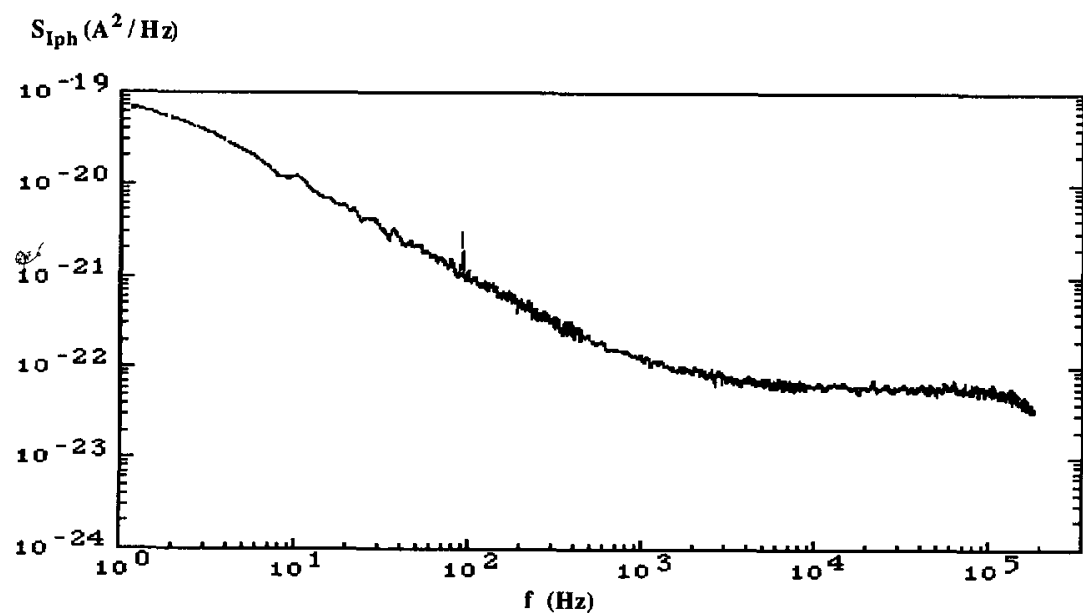

b)

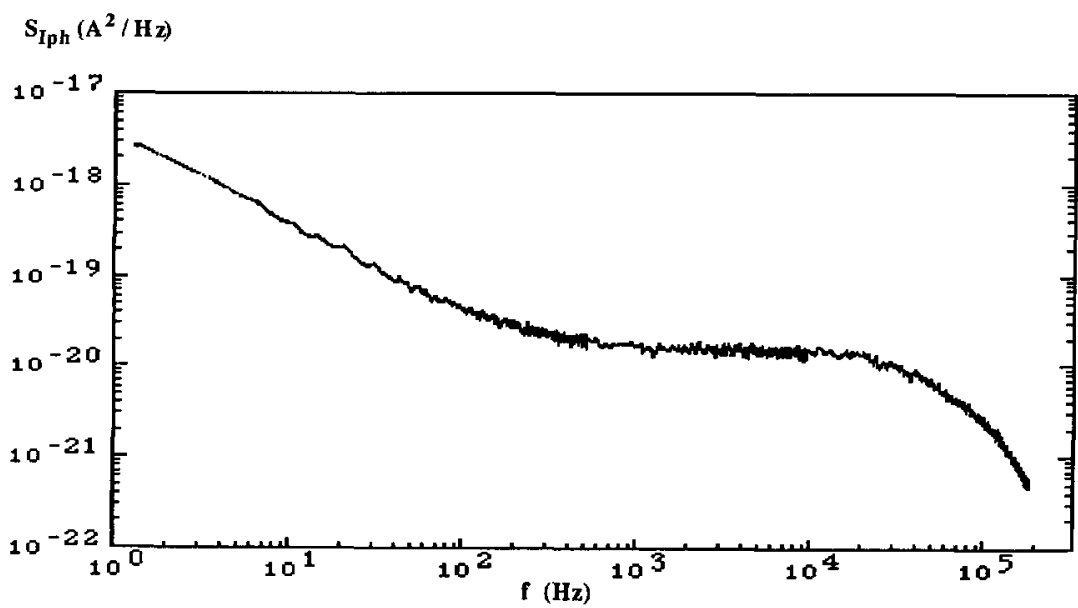

c)

Fig. 7. - Spectres en fréquence du Bruit Optique dans les 2 configurations $(\alpha)$ et $(\beta)$. [Optical spectral density versus frequency in both configurations $(\alpha)$ and $(\beta)$.] 
Sur la figure 7c qui présente le spectre en fréquence du bruit optique en configuration $(\beta)$ sous un courant de polarisation de 1,3 fois le courant de seuil, nous constatons une évolution typiquement Lorentzienne : "plateau de bruit" suivi d'une décroissance en $1 / f^{\gamma}$, avec $1 \leq \gamma \leq 2$. Un tel comportement est attribué à un phénomène de sauts de modes optiques, souvent désigné par le terme "mode hopping" [8] : il y a compétition modale entre le mode de Bragg intrinsèque à la structure DFB et un mode résiduel Fabry-Pérot parasite, "image" optique du mode de Bragg. Ces modes échangent de l'énergie de manière aléatoire, d'où un niveau de bruit très élevé.

Cette compétition modale et son bruit associé ont été observés par plusieurs auteurs $[9,10]$. Le saut de modes optiques engendre des fluctuations très importantes du nombre de porteurs et de photons dans la cavité optique $[9,10]$, ces dernières étant directement responsables des fluctuations de tension observées aux bornes de la diode laser et aussi des fluctuations de la puissance optique émise par le laser.

Notons enfin que la présence d'un tel phénomène engendre une dépendance temporelle en "créneaux" des tensions et courants de bruit, respectivement $V_{\mathrm{d}}(t)$ et $I_{\mathrm{ph}}(t)$; cette dépendance a été également observée par Ohtsu et al. [4]. Nous avons aussi constaté un tel comportement pour des lasers à puits quantique [2].

Cette plage critique dans laquelle nous observons une compétition modale s'étend environ de 1,1 à 1,5 fois le courant de seuil. Au delà, le spectre de bruit optique en fréquence retrouve l'allure normale : bruit en $1 / f$ puis bruit blanc.

Nous obtenons également une évolution Lorentzienne, sous les mêmes conditions, pour la densité spectrale de bruit électrique [1].

4.2.2. Densités spectrales fonction du courant laser $I_{\text {Laser }}$ injecté - fonction du photocourant $I_{\mathrm{ph}}$ détecté

4.2.2.1. Configuration optique $(\alpha)$

i) La densité spectrale de bruit blanc optique $S_{I_{\mathrm{ph}}}$ et le bruit d'intensité relative $R I N$ mesurés à $200 \mathrm{kHz}$ sont représentés sur la figure $8 \mathrm{a}$.

Le RIN représente, par définition, les fluctuations relatives du photocourant détecté :

$$
R I N(f)=\frac{S_{I_{\mathrm{ph}}}(f)-2 q I_{\mathrm{ph}}}{I_{\mathrm{ph}}^{2}}
$$

la densité spectrale $S_{I_{\mathrm{ph}}}$ est étalonnée par rapport au bruit de grenaille $2 q I_{\mathrm{ph}}$ propre au photodétecteur.

- Pour des photocourants $I_{\mathrm{ph}}$ détectés inférieurs à $1,5 \mu \mathrm{A}$, correspondant à des courants de polarisation $I_{\text {Laser }}$ inférieurs à $0,85 I_{\text {seuil }}$, donc en régime d'émission spontanée, le bruit blanc optique mesuré est égal au bruit de grenaille du photodétecteur, soit $2 q I_{\mathrm{ph}}$.

- Dans la région de superluminescence, la densité spectrale $S_{I_{\mathrm{ph}}}$ augmente rapidement avec le courant injecté.

- Peu après, en régime laser, le bruit sature légèrement, puis ré-augmente à nouveau avec une dépendance en $I_{\mathrm{ph}}$ voisine de $I_{\mathrm{ph}}^{+0,2}$, pour $I_{\text {Laser }} / I_{\text {seuil }} \geq 1,05$.

Pour les diodes laser $\mathrm{DFB}$, en régime d'émission stimulée le $R I N$ mesuré décroît généralement en $I_{\mathrm{ph}}^{-3}[11]$. Ce n'est pas ce que nous observons ici dans cette configuration optique. L'augmentation, en régime laser, de la densité spectrale $S_{I_{\mathrm{ph}}}$ mesurée ici peut être due à une augmentation du bruit propre au mode "lasant" : le spectre optique correspondant vu au paragraphe précédent se partageait en effet en un mode principal et deux sous modes latéraux, pour des courants injectés supérieurs à 2,5 fois le courant de seuil.

ii) Le bruit électrique, traduisant les fluctuations de la tension $V_{\mathrm{d}}$ aux bornes de la diode laser, est caractérisé par les densités spectrales exprimées en tension de bruit: $S_{V_{\mathrm{d}}}\left(\mathrm{en} \mathrm{V}^{2} / \mathrm{Hz}\right)$ 


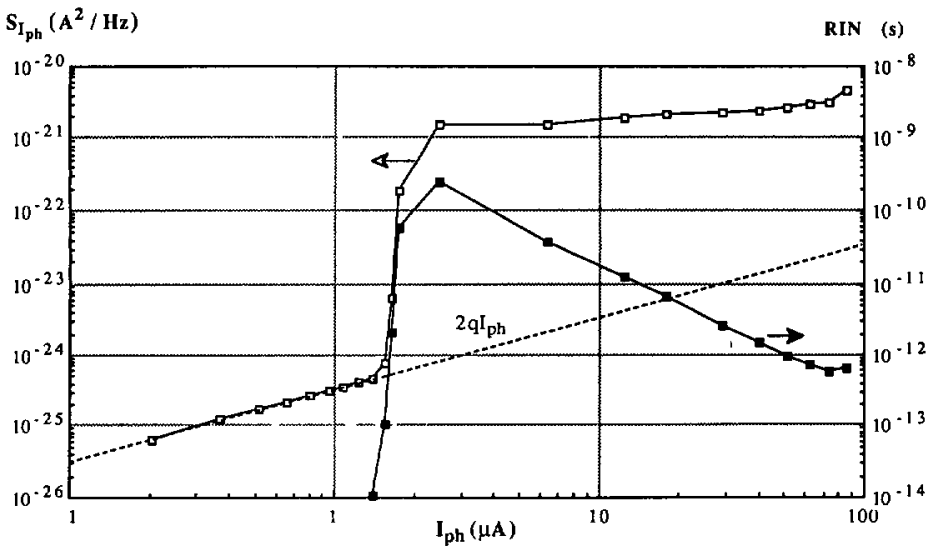

a)

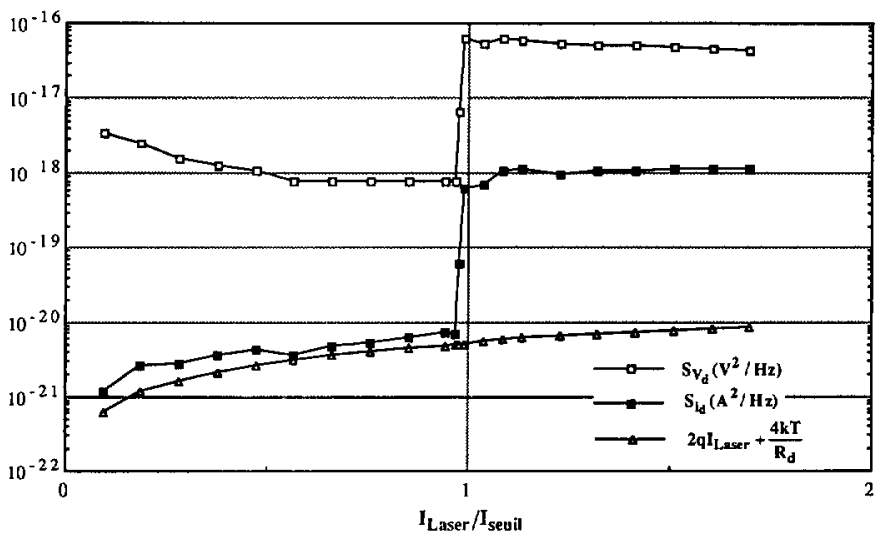

b)

Fig. 8. - Evolution des densités spectrales de bruit électrique et optique mesurées à $200 \mathrm{kHz}$ en configuration $(\alpha)$.

[Optical and electrical spectral densities measured at $200 \mathrm{kHz}$ in configuration $(\alpha)$.]

ou en courant de bruit: $S_{\imath_{\mathrm{d}}}\left(\mathrm{en} \mathrm{A}^{2} / \mathrm{Hz}\right)$. Ces dernières sont reliées par la loi d'Ohm :

$$
S_{\mathrm{id}_{\mathrm{d}}}=\frac{S_{V_{\mathrm{d}}}}{R_{\mathrm{d}}^{2}}
$$

où $R_{\mathrm{d}}$ désigne la résistance différentielle introduite au paragraphe 2.2 .

En examinant la figure 8b, nous constatons tout d'abord que la densité spectrale $S_{\imath_{\mathrm{d}}}$ excède la somme du bruit de grenaille $\left(2 q I_{\text {Laser }}\right)$ et du bruit thermique lié à la résistance série $\left(4 k T / R_{s}\right)$, indiquant ainsi que le bruit électrique mesuré à $200 \mathrm{kHz}$ en régime d'émission laser est propre à la jonction émettrice.

- A l'approche du seuil, les deux densités $S_{V_{\mathrm{d}}}$ et $S_{\imath_{\mathrm{d}}}$ augmentent de manière très importante et le niveau atteint se situe environ deux décades au-dessus du niveau observé juste avant le seuil. Un tel comportement en bruit électrique au voisinage du seuil a été observé et également calculé pour des diodes laser Fabry-Pérot [12].

- Par contre, le comportement en fort régime d'émission stimulée n'est pas du tout "classique" : Ia densité en courant $S_{\imath_{\mathrm{d}}}$ ne rejoint jamais ici la somme du bruit de grenaille et du bruit thermique, mais reste pratiquement constante. 


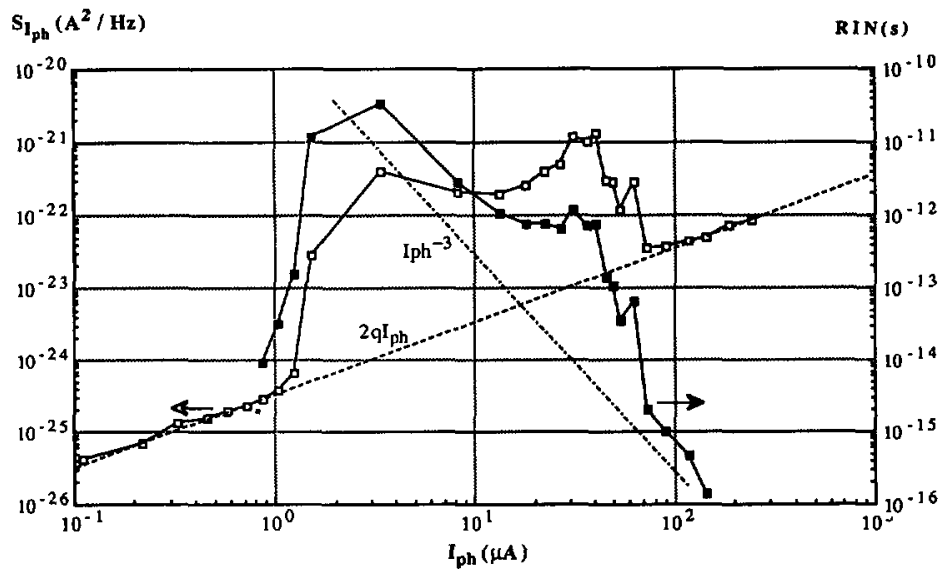

a)

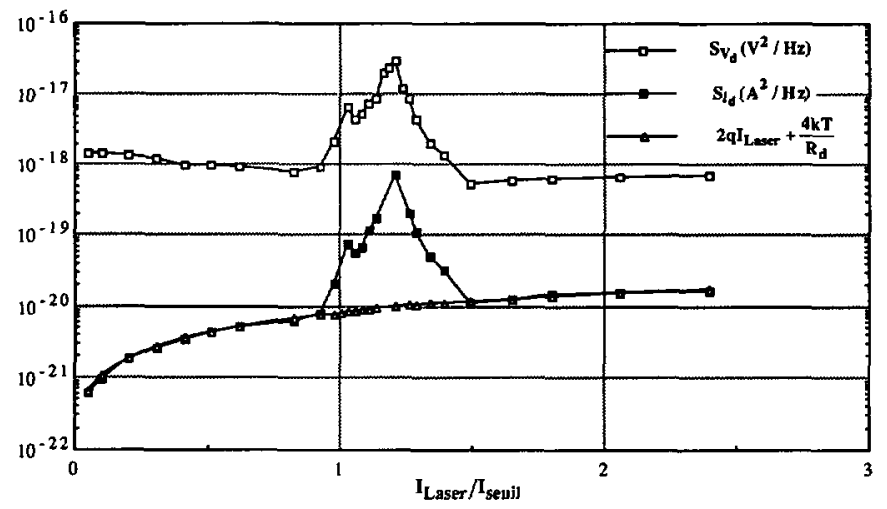

b)

Fig. 9. - Evolution des densités spectrales de bruit électrique et optique mesurées à $200 \mathrm{kHz}$ en configuration $(\beta)$.

[Optical and electrical spectral densities measured at $200 \mathrm{kHz}$ in configuration $(\beta)$.]

Nous observions le même comportement avec le bruit optique, sous les mêmes conditions : cette similitude entre bruits électrique et optique est un point essentiel de cette étude.

\subsubsection{Configuration optique $(\beta)$}

i) L'évolution avec le photocourant détecté du bruit blanc optique et du $R I N$ mesurés après un reclivage de la fibre optique est donnée figure $9 \mathrm{a}$.

- En dessous du seuil et en superluminescence, le comportement est tout à fait similaire à celui observé précédemment en situation $(\alpha)$.

- Par contre, en faible régime laser, soit pour $I_{\mathrm{ph}}$ compris entre 3 et $10 \mu \mathrm{A}$, le niveau de bruit optique blanc diminue et tend vers le bruit de grenaille $2 q I_{\mathrm{ph}}$. Le $R I N$ décroît avec une pente avoisinant les $I_{\mathrm{ph}}^{-3}$, conformément aux résultats obtenus par Yoshikuni [11] et Joindot [13].

- Au delà, le bruit optique ré-augmente d'environ une décade ; cette croissance ainsi que l'allure en "dents de scie" observée sont probablement dues au phénomène de saut de modes évoqué au paragraphe précédent : il y a une forte compétition modale entre le mode fondamental de Bragg, intrinsèque à la structure DFB, et le mode parasite Fabry-Pérot, "image" optique du mode de Bragg.

- En fort régime d'émission stimulée, pour $I_{\mathrm{ph}}>70 \mu \mathrm{A}$, la densité spectrale optique $S_{I_{\mathrm{ph}}}$ tend sensiblement vers le niveau de bruit de grenaille de la photodiode. 
ii) Examinons maintenant l'évolution du bruit électrique.

- En régime d'émission spontanée, celui-ci est identique dans les situations $(\alpha)$ et $(\beta)$ : comparer les figures $8 \mathrm{~b}$ et $9 \mathrm{~b}$.

- A l'approche du seuil laser, les densités spectrales $S_{V_{\mathrm{d}}}$ et $S_{\imath_{\mathrm{d}}}$ augmentent très rapidement.

On observe ensuite une légère décroissance suivie d'une brusque ré-augmentation. L'allure en dents de scie enregistrée, tout à fait semblable à celle de la densité de bruit optique sous les mêmes conditions, est caractéristique du phénomène de compétition modale [9].

- Au delà, le bruit électrique exprimé en courant de bruit, soit $S_{\imath_{\mathrm{d}}}$, tend vers le bruit de grenaille lié au courant moyen injecté.

Il est enfin intéressant de noter que les maxima des bruits électrique et optique se situent exactement aux mêmes courants de polarisation : comparer les figures $8 \mathrm{~b}$ et $9 \mathrm{~b}$. Ceci indique que les deux bruits, pourtant de nature différente, sont étroitement liés.

En résumé, la caractérisation par la mesure des densités spectrales de bruit blanc permet de mettre en évidence et de différencier deux phénomènes parasites de réinjection optique.

Le premier apparaît en configuration $(\alpha)$, c'est à dire en présence d'une réinjection parasite externe à la tête optique, et induit du bruit de partition : ce bruit est lié à une fluctuation de la répartition d'énergie entre le mode principal et ses sous modes. Ce processus bien décrit dans la littérature [3] se produit pratiquement sur toute la plage d'émission stimulée.

Quant au phénomène de compétition modale observé en situation $(\beta)$, compétition entre le mode fondamental de Bragg et un mode résiduel Fabry-Pérot, il n'apparaît que dans une plage réduite de courants de polarisation en régime d'émission laser.

4.3. INTERPRÉtATION - CONFRONTATION EXPÉRIENCE - THÉORIE. - Le courant de polarisation $I$ injecté dans la zone active alimente celle-ci en porteurs. Soit ceux-ci se recombinent spontanément avec une durée de vie $\tau_{\text {nsp }}$ et alors une fraction $\beta$ de ces recombinaisons fournit des photons au mode laser. Soit ces porteurs donnent naissance au gain $G$ nécessaire pour l'émission stimulée ( $G=E_{\mathrm{cv}}-E_{\mathrm{vc}}$, taux net d'émission stimulée) : il y a alors formation de photons et déclenchement du phénomène d'amplification dans la zone active. Certains photons sont absorbés, d'autres sont émis par la diode laser et contribuent ainsi à la puissance optique en sortie. La quantité $\tau_{\mathrm{ph}}$ désignera la durée de vie moyenne des photons dans le milieu amplificateur.

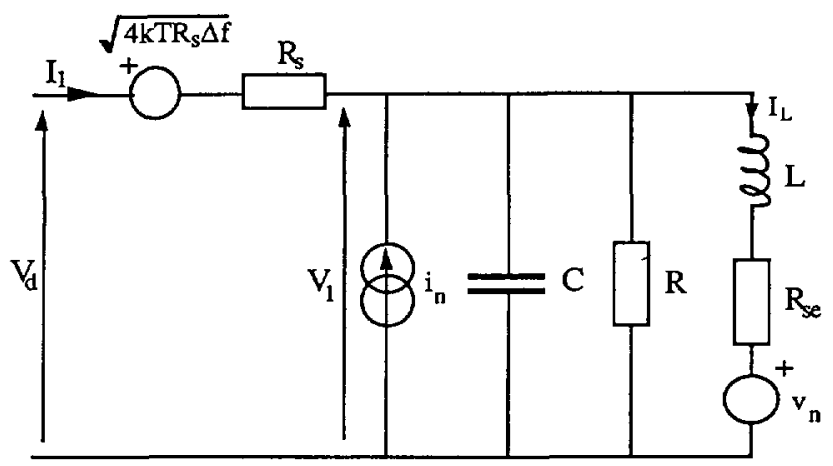

Fig. 10. - Schéma équivalent de bruit du laser monomode.

[Noise equivalent circuit of a monomode laser diode.] 
En se basant sur cette suite de processus, nous pouvons écrire les deux équations d'état qui régissent la dépendance temporelle du nombre de porteurs $n$ et du nombre de photons $s$ :

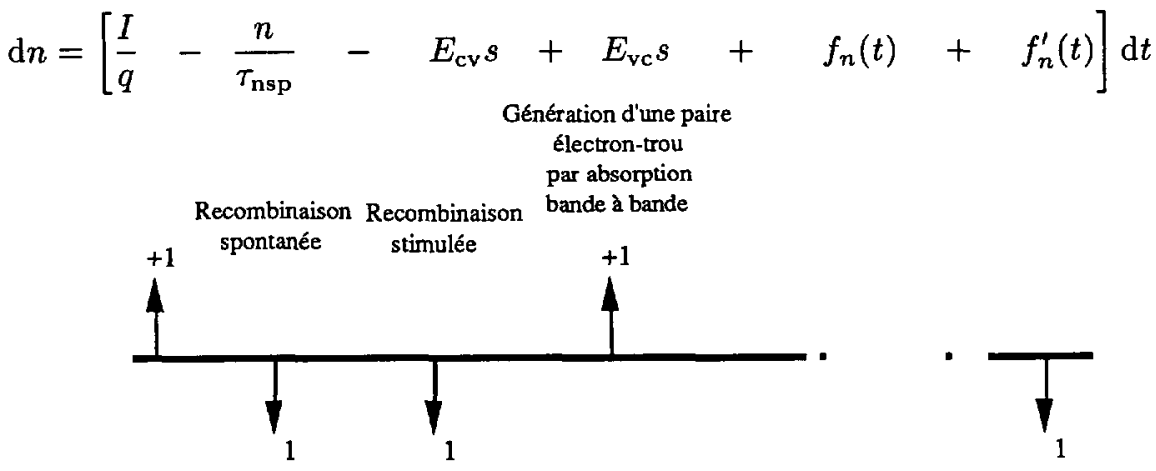

$$
\begin{aligned}
& \mathrm{d} s=\left[\beta \frac{n}{\tau_{\mathrm{nsp}}}+E_{\mathrm{cv}} s-E_{\mathrm{vc}} s-\frac{s}{\tau_{\mathrm{ph}}}+f_{s}(t)+f_{s}^{\prime}(t)\right] \mathrm{d} t \\
& \text { Emission spontanée } \\
& \text { couplée au mode } \\
& \text { oscillant du laser Emission stimulée } \\
& \text { (1) Absorption Emission optique }
\end{aligned}
$$

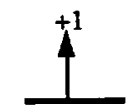

Les flèches illustrent les variations instantanées aléatoires du nombre d'électrons $\mathrm{d} n$ et de photons $\mathrm{d} s$, égales à +1 ou -1 . Les sources de bruit blanc $f_{n}$ et $f_{s}$ données par le modèle de Mc Cumber [14] expriment respectivement la distribution aléatoire des instants de génération-recombinaison de porteurs et la distribution des instants d'émission et d'absorption des photons.

Dans le cas d'une réinjection optique, la présence d'un paquet de photons retournant vers la cavité émettrice rajoute une contribution, soit $f_{s}^{\prime}$, par variation instantanée de la population de photons ; cette contribution engendre directement une source $f_{n}^{\prime}$ puisque les deux populations porteurs-photons sont étroitement liées.

Mais dans ce cas, il faudrait également tenir compte de la phase de l'onde retour, donc introduire une troisième équation, reliant les fluctuations d'amplitude et celles de la phase : nous réservons cette étude à des travaux ultérieurs.

En appliquant le régime de petits signaux aux équations précédentes, nous obtenons le schéma équivalent de bruit donné figure $10[1,12]$ et valable en l'absence de réinjection optique.

$i_{n}$ et $v_{n}$ indiquent respectivement, la source de courant traduisant les fluctuations du nombre de porteurs et la source de tension reliée aux fluctuations du nombre de photons du mode laser. Les autres éléments appartenant au circuit sont décrits avec précision dans [12] et [1].

Ainsi, les résultats expérimentaux en bruit exposés au paragraphe 4.2 peuvent être interprétés à l'aide du schéma équivalent de bruit d'un laser monomode, en incluant aux équations classiques d'évolution les sources de bruit blanc de Langevin. 
4.3.1. Expression théorique du $R I N$. - Du schéma précédent, nous déduisons l'expression du bruit blanc d'intensité relative $R I N$ :

$$
R I N \approx \frac{R^{2}\left\{S_{i_{n}}-\frac{2}{R} S_{\imath_{n} v_{n}}+\frac{1}{R^{2}} S_{v_{n}}\right\}}{(q s G)^{2}\left(R+R_{\mathrm{se}}\right)^{2}}
$$

- La densité spectrale de courant de bruit $S_{\imath_{n}}$ représente les fluctuations de la population d'électrons. Cette densité traduit le bruit de grenaille dû au courant moyen injecté en régime spontané et représente le bruit supplémentaire lié à l'absorption de photons dans le milieu actif en régime laser.

- La densité spectrale de tension de bruit $S_{v_{n}}$ traduit les fluctuations de la population de photons. Lorsque le nombre de porteurs injectés augmente, donc lorsque le gain sature, cette fluctuation diminue puis tend à devenir constante.

- Enfin, la source croisée de courant-tension de bruit $S_{2_{n} v_{n}}$ représente l'influence réciproque des fluctuations de populations d'électrons $n$ et de photons $s$. Cette source est pratiquement constante en régime laser puisqu'alors inversement proportionnelle au nombre moyen de porteurs injectés.

En régime laser, compte-tenu des ordres de grandeur respectifs des différentes sources de bruit intrinsèques précitées, l'équation précédente se simplifie :

$$
R I N_{I>I_{\text {seutl }}} \approx \frac{S_{v_{n}}}{(q s G)^{2} R^{2}}
$$

Nous voyons donc ainsi apparaître la prédominance du terme $S_{v_{n}}$, traduisant les fluctuations de la population de photons.

D'autre part, cette source $S_{v_{n}}$ tend en régime laser à être constante : le $R I N$ est donc alors inversement proportionnel à la population de photons $s$.

En fort régime d'émission stimulée, soit pour $I_{\text {Laser }}>2 I_{\text {seuil }}$, c'est à dire lorsque le nombre moyen $s_{0}$ de photons émis devient supérieur à $10^{5}$, l'expression théorique du bruit $R I N$ blanc se simplifie encore et s'exprime simplement sous la forme d'une fraction inversement proportionnelle au courant injecté :

$$
R I N_{I>2 I_{\text {seul }}} \approx \frac{4 q\left(1+\tau_{\mathrm{ph}} E_{\mathrm{vc}}\right)}{I_{\text {Laser }}-I_{\text {seuil }}}
$$

4.3.2. Expression théorique de la densité spectrale $S_{V_{\mathrm{d}}}$ de bruit électrique. - En basse et moyenne fréquence l'expression du bruit électrique est :

$$
S_{V_{\mathrm{d}}}(\omega)=4 k T R_{\mathrm{s}}+S_{V_{1}}(\omega)
$$

où le premier terme $4 k T R_{\mathrm{s}}$ traduit le bruit thermique lié à la résistance série $R_{\mathrm{s}}$.

$S_{V_{1}}$ désigne la densité spectrale de fluctuations de type blanc de la tension intrinsèque à la jonction laser.

$$
S_{V_{1}} \approx \frac{R^{2}\left(R_{\mathrm{se}}^{2} S_{2_{n}}+2 R_{\mathrm{se}} S_{v_{n} v_{n}}+S_{v_{n}}\right)}{\left(R+R_{\mathrm{se}}\right)^{2}}
$$

En régime laser, cette expression se simplifie considérablement et nous obtenons :

$$
S_{V_{\mathrm{d}_{I}>I_{\text {seul }}}} \approx 4 k T R_{s}+S_{v_{n}}
$$

Nous constatons ainsi à nouveau la prédominance, en régime d'émission stimulée, de la fluctuation de la population de photons exprimée par le biais du terme $S_{v_{n}}$.

La similitude constatée expérimentalement entre les deux densités spectrales de bruit, électrique et optique, apparaît donc tout à fait clairement grâce à ce développement théorique. 


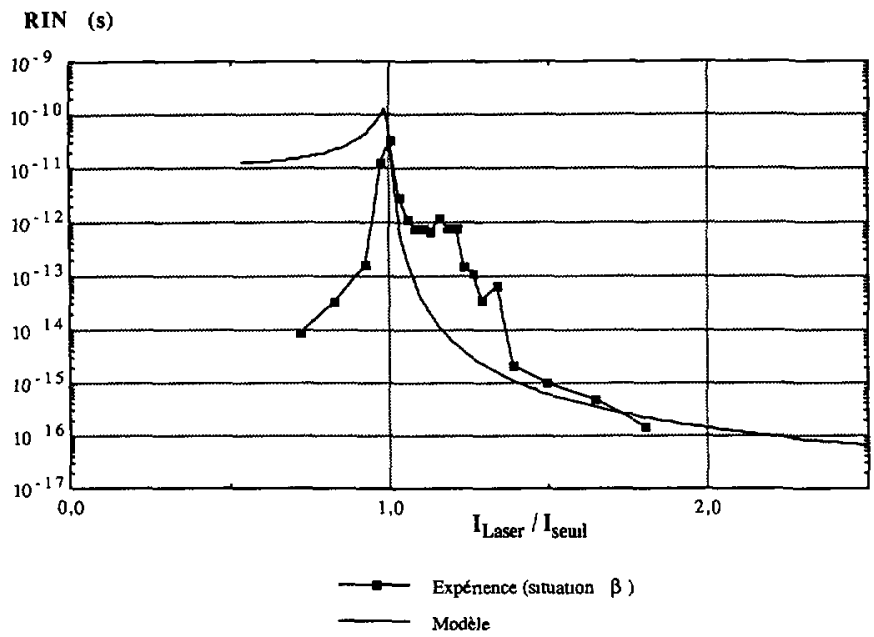

Fig. 11. - Comparaison des Bruits Blancs d'Intensité Relative, théorique et expérimental. [Comparison of theoretical and experimental Relative Intensity Noises.]

4.3.3. Confrontation expérience-théorie. - Sur la figure 11 sont comparés les $R I N$ expérimental, obtenu après minimisation des réflexions parasites externes c'est à dire en configuration $(\beta)$, et théorique.

Nous constatons que le modèle théorique développé n'est avant tout applicable qu'en régime d'émission laser.

En effet, en régime d'émission spontanée, le niveau de bruit blanc théorique est beaucoup plus élevé que le niveau mesuré : cette différence peut être liée à une surestimation, dans cette plage de polarisation, de la densité spectrale de fluctuations de la source de Langevin $f_{s}(t)$.

En faible régime d'émission laser, apparaît expérimentalement une remontée du niveau de bruit optique : nous avons vu que ce phénomène est lié à une compétition modale entre le mode de Bragg, intrinsèque à la structure DFB, et un mode Fabry-Pérot parasite, "image" optique du précédent. Ce comportement physique est délicat à traduire de manière quantitative : nous réservons cette étude pour des travaux ultérieurs.

Examinons maintenant la figure 12, où sont comparés les bruits électriques expérimental, obtenu en situation $(\beta)$, et théorique. Nous constatons un accord satisfaisant entre les deux caractéristiques. Toutefois, en fort régime d'émission laser, le modèle développé prévoit un niveau décroissant avec le courant injecté et devenant inférieur au niveau mesuré, qui est pratiquement constant. De plus, compte-tenu que le niveau de bruit électrique blanc $S_{V_{\mathrm{d}}}$ mesuré tend, en fort régime stimulé, vers la quantité $4 k T R_{s}+R_{s}^{2} 2 q I_{\text {Laser }}$ (Fig. $9 \mathrm{~b}$ ), il apparaît que le modèle prévoit un comportement "sous-Poissonien" - la densité spectrale intrinsèque $S_{v_{n}}$ devient inférieure au terme traduisant le bruit de grenaille de la jonction émettrice traversée par le courant $I_{\text {Laser }}$, à savoir $R_{s}^{2} 2 q I_{\text {Laser }}$.

\section{Corrélation électrique-optique à $200 \mathrm{kHz}$}

La fonction de corrélation électrique-optique s'écrit par définition :

$$
\gamma_{I_{\mathrm{ph}}-V_{\mathrm{d}}}^{2}(f)=\frac{\left|S_{I_{\mathrm{ph}}-V_{\mathrm{d}}}(f)\right|^{2}}{S_{I_{\mathrm{ph}}}(f) S_{V_{\mathrm{d}}}(f)} \quad \text { avec } \quad 0 \leq \gamma_{I_{\mathrm{ph}}-V_{\mathrm{d}}}^{2}(f) \leq 1
$$




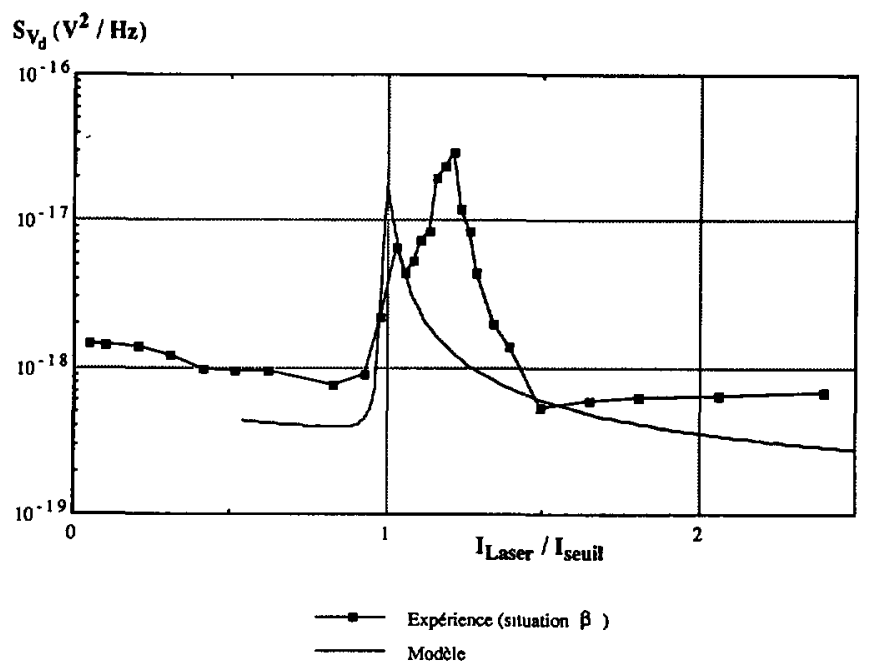

Fig. 12. - Comparaison des Bruits Electriques Blancs, théorique et expérimental.

[Comparison of theoretical and experimental Electrical Spectral Densities.]

où $S_{I_{\mathrm{ph}}-V_{\mathrm{d}}}(f)$ représente la densité spectrale d'intercorrélation entre bruit optique $S_{I_{\mathrm{ph}}}$ et bruit électrique $S_{V_{\mathrm{d}}}$. La corrélation dans l'espace des fréquences indique le degré de dépendance d'un signal par rapport à un autre.

5.1. RÉSultats EXPÉRIMENTAuX À $200 \mathrm{kHz}$. - Sur la figure 13a,b est donnée l'évolution, avec le courant d'injection rapporté au courant de seuil, de la fonction de corrélation mesurée à $200 \mathrm{kHz}$, respectivement en situation $(\alpha)$ et $(\beta)$.

- En régime d'émission spontanée, il est naturel de trouver une corrélation nulle puisque le bruit optique mesuré dans cette région est largement dominé par le bruit de grenaille du photodétecteur.

- Dans la région de superluminescence, la cohérence augmente très rapidement, de même que les densités spectrales de bruit électrique et optique, et atteint pratiquement l'unité. Le maximum observé peut être expliqué par de fortes fluctuations du gain optique, au voisinage du seuil, en très faible régime laser [14].

- Au delà et en configuration $(\alpha)$, la corrélation décroît lentement en restant toujours très élevée. Cette évolution reflète bien celle des bruits optique et électrique : le niveau des deux densités spectrales $S_{I_{\mathrm{ph}}}$ et $S_{V_{\mathrm{d}}}$ reste, en émission stimulée, très élevé mais $S_{I_{\mathrm{ph}}}$ continue à croître alors que $S_{V_{d}}$ décroît légèrement, d'où la lente "décorrélation" observée.

- Par contre, en configuration $(\beta)$, la corrélation mesurée à $200 \mathrm{kHz}$ tend rapidement vers zéro : figure 13b. En fort régime laser, le bruit optique mesuré tend, dans cette configuration, vers le bruit de grenaille du photodétecteur ; il est donc logique de mesurer une corrélation électrique-optique nulle. Quant au phénomène de compétition modale évoqué dans les paragraphes précédents, il engendre une forte remontée du niveau de corrélation, toujours dans la même plage de polarisation, à savoir entre 1,2 et 1,3 fois le courant de seuil.

Il apparaît ainsi que la présence d'une source de bruit prédominante engendre, quel que soit sa nature, un niveau de corrélation électrique-optique fort et d'autant plus élevé que cette source est majoritaire.

La fonction de corrélation s'avère donc être un outil, à la fois très fin et très puissant, d'analyse du comportement en bruit. 


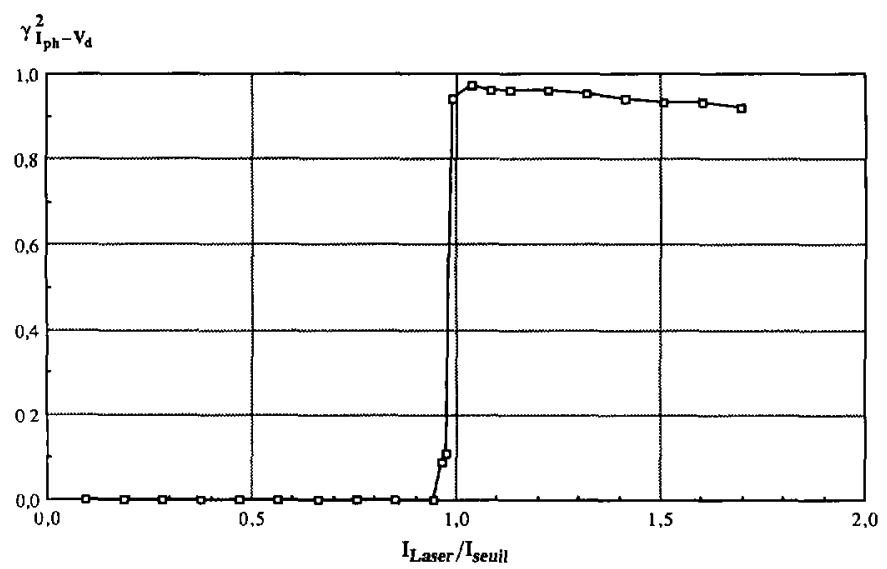

a)

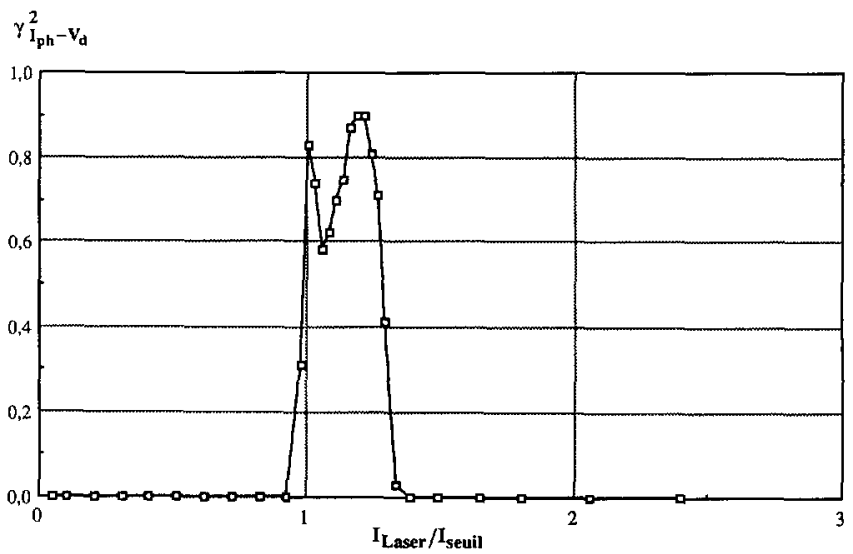

b)

Fig. 13. - Evolution avec le courant laser de la fonction de corrélation dans les deux configurations. [Electrical-optical correlation measured at $200 \mathrm{kHz}$ in both configurations.]

\subsection{EXPRESSION THÉORIQUE DE LA FONCTION DE CORRÉLATION ÉLECTRIQUE-OPTIQUE}

De part le schéma équivalent présenté précédemment, nous pouvons établir l'expression théorique de la fonction de corrélation à $200 \mathrm{kHz}$ à partir des sources de bruit intrinsèques, c'est à dire $S_{\imath_{n}}, S_{v_{n}}$ et $S_{\imath_{n} v_{n}}$. L'expression obtenue est très lourde et peu exploitable.

Toutefois, compte-tenu de l'importance, principalement en régime d'émission laser, de la source de bruit intrinsèque $S_{v_{n}}$, certaines simplifications sont permises. Il vient alors [1] :

$$
\gamma_{I_{\mathrm{ph}}-V_{\mathrm{d}}}^{2}(\omega) \approx \frac{1}{1+\frac{4 k T R_{\mathrm{s}}}{S_{V_{1}}(\omega)}}
$$

Ainsi, la corrélation électrique-optique n'est fonction que de la densité spectrale $S_{V_{1}}$ de fluctuations de la tension $V_{1}$. La relation précédente permet d'évaluer directement à partir du niveau de bruit blanc électrique mesuré la fonction de corrélation entre les deux sources de bruit, électrique et optique.

5.3. EXPRESSION THÉORIQUE COMPLÈTE DE LA FONCTION DE CORRÉLATION. - Il est nécessaire, pour plus de rigueur, de prendre en compte les bruits générés par les différents éléments 


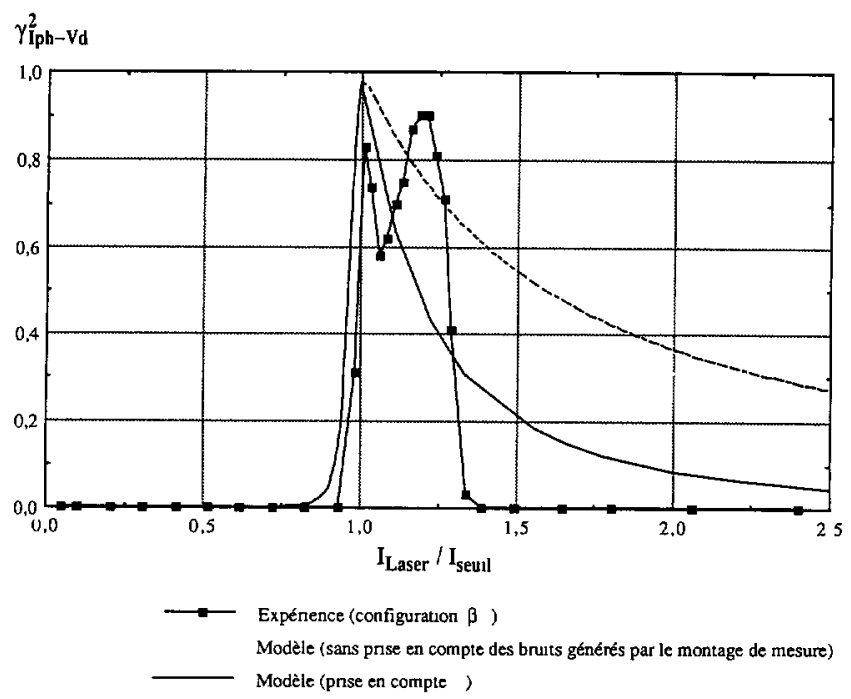

Fig. 14. - Comparaison des fonctions de corrélation électrique-optique, théoriques et expérimentales. [Comparison of theoretical and experimental Electrical-Optical Correlations.]

qui composent le banc de mesure tels que les amplificateurs de tension et de courant sans oublier le photodétecteur. Chacune des différentes sources de bruit étant décorrélée une à une, les densités spectrales de bruit réellement mesurées par l'analyseur sont égales à la somme des différentes densités spectrales.

$$
\text { Ainsi } \quad\left\{\begin{array}{l}
S_{I_{\mathrm{ph}}-V_{\mathrm{d}}}(f)=a(\omega) S_{V_{1}}(f) \\
S_{I_{\mathrm{ph}}}(f)=|a(\omega)|^{2} S_{V_{1}}(f)+S_{\imath_{\mathrm{a}}}(f)+S_{i_{\mathrm{g}}}(f) \\
S_{V_{\mathrm{d}}}(f)=S_{V_{1}}(f)+S_{u_{\mathrm{n}}}(f)+S_{v_{\mathrm{a}}}(f)
\end{array} \quad(12 \mathrm{a}, \mathrm{b}, \mathrm{c})\right.
$$

avec $\quad S_{\imath_{\mathrm{a}}}(f) \quad$ bruit de l'amplificateur de courant

$S_{\imath_{\mathrm{g}}}(f) \quad$ bruit de grenaille du photodétecteur

$S_{u_{\mathrm{n}}}(f) \quad$ bruit thermique lié à la résistance série $R_{\mathrm{s}}$

$S_{v_{\mathrm{a}}}(f) \quad$ bruit de l'amplificateur de tension

et

$$
a(\omega) \approx \frac{\sigma h v}{\tau_{\mathrm{ph}}} \frac{1}{q G} \frac{R_{\mathrm{se}} / R}{R_{\mathrm{se}}+\mathrm{j} L \omega} \quad[1] \quad \text { en } \mathrm{Ohms}^{-1}
$$

$\sigma$ désigne la sensibilité apparente, incluant la sensibilité du photodétecteur et également les pertes dues aux différents couplages.

Remarque : la densité spectrale $S_{V_{1}}$ intervient dans les trois équations théoriques (12), d'où la corrélation électrique-optique constatée.

5.4. Confrontation. - Sur la figure 14 sont comparées les fonctions de corrélation électriqueoptique théoriques, obtenues avec et sans prise en compte des bruits générés par le montage de mesure, et expérimentale, mesurée en configuration $(\beta)$, donc après minimisation des réflexions parasites externes au boîtier laser.

Il apparaît ainsi clairement que les bruits propres au montage de mesure engendrent, ce qui est logique, une brusque et forte "décorrélation" entre les deux densités spectrales de bruit, électrique et optique. 


\section{Conclusion}

Les mesures, dans une plage de moyenne fréquence $(10 \mathrm{kHz}-200 \mathrm{kHz})$, des densités spectrales de bruit électrique et optique d'une tête d'émission laser DFB permettent la mise en évidence et la différenciation de deux phénomènes parasites optiques : le processus de fluctuation de l'énergie lumineuse qui engendre un bruit en excès dit bruit de partition et un phénomène de compétition modale entre le mode de Bragg et un mode résiduel Fabry-Pérot.

Cette caractérisation par le bruit confirme également une fois de plus le lien étroit existant entre les deux densités spectrales, électrique et optique : le bruit électrique peut ainsi être utilisé à lui seul pour une caractérisation in situ du composant, sans mise en oeuvre d'un montage optique délicat et source de réflexions parasites vers la cavité laser.

Enfin, il apparaît clairement dans cette étude que la fonction de corrélation est un outil extrêmement fin d'analyse du comportement en bruit : la présence d'une source de bruit prédominante engendre une remontée du niveau de corrélation électrique-optique, remontée d'autant plus forte que cette source est majoritaire.

\section{Bibliographie}

[1] Signoret P., Analyse des Bruits Optique-Electrique et étude de leur corrélation - Application à une tête optique d'émission laser à réaction répartie, Thèse Ph.D. (Université Montpellier II, 1994).

[2] Orsal B., Peransin J.M., Signoret P. et Daulasim K., Low frequency Noise of a $980 \mathrm{~nm}$ InGaAs/GaAs Strained Quantum Well Laser, J. Phys. III, France 3 (1993) 1739-1749.

[3] Lenstra D. et Cohen J.S., Feedback noise in single mode semiconductor lasers, Laser Noise, Proc. SPIE 1376 (1990) 245-258.

[4] Ohtsu M., Analyses of mode partition and mode hopping in semiconductor lasers, IEEE J. Quantum Electron. 25 (1989) 31-38.

[5] Kazarinov R.F. et Henry C.H., The relation of line narrowing and chirp reduction resulting from the coupling of a semiconductor laser to a passive resonator, IEEE J. Quantum Electron. 23 (1987) 1401-1409.

[6] Temkin H. et al., Reflection noise in index guided InGaAsP lasers, IEEE J. Quantum Electron. 22 (1986) 286-293.

[7] Spano P. et al., Theory of noise in semiconductor lasers in the presence of optical feedback, IEEE J. Quantum Electron. 20 (1984) 350-357.

[8] Yamada M., Theory of mode competition noise in semiconductor injection lasers, IEEE J. Quantum Electron. 22 (1986) 1052-1059.

[9] Andrekson P.A., Andersson P. et Alping A., Electrical noise measurements on laser diodes for monitoring of optical feedback and mode hopping, Electron. Lett. 22 (1986) 195-196.

[10] Chinone N., Kuroda T., Ohtoshi T., Takahashi T, et Kajimura T., Mode hopping noise in index guided semiconductor lasers and its reduction by saturable absorbers, IEEE J. Quantum Electron. 21 (1985) 1264-1270.

[11] Yoshikuni Y. et al., Intensity fluctuations of $1.5 \mu \mathrm{m}$ InGaAsP/InP Distributed Feedback Lasers involving the optical feedback effect, IEE Proc. 132 (1985) 20-27.

[12] Harder C., Katz J., Margalit S., Schacham J.S. et Yariv A., Noise equivalent circuit of a semiconductor laser diode, IEEE J. Quantum Electron. 18 (1982) 333-337.

[13] Joindot I., Measurements of Relative Intensity Noise (RIN) in semiconductor lasers, J. Phys. III, France 2 (1992) 1591-1603.

[14] Cumber Mc, Intensity fluctuations in the output of C.W. laser oscillations, Phys. Rev. 141 (1966) 306-322. 